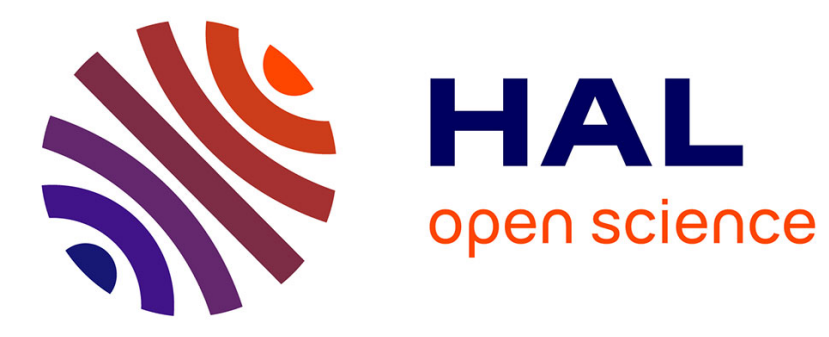

\title{
Microwave-optical fiber lasers stabilized by frequency-shifted feedback
}

\author{
Marie Guionie, Aurélien Thorette, Marco Romanelli, Anthony Carré, \\ Goulc'Hen Loas, Emmanuel Pinsard, Laurent Lablonde, Benoît Cadier, Mehdi
} Alouini, Maxime Vallet, et al.

\section{To cite this version:}

Marie Guionie, Aurélien Thorette, Marco Romanelli, Anthony Carré, Goulc'Hen Loas, et al.. Microwave-optical fiber lasers stabilized by frequency-shifted feedback. 2019 IEEE International Topical Meeting on Microwave Photonics (MWP 2019), Oct 2019, Ottawa, Canada. IEEE, IEEE Xplore Digital Library, pp.1-4, 2019, 2019 International Topical Meeting on Microwave Photonics (MWP). 10.1109/MWP.2019.8892036 . hal-02470792

\section{HAL Id: hal-02470792 https://hal.science/hal-02470792}

Submitted on 7 Feb 2020

HAL is a multi-disciplinary open access archive for the deposit and dissemination of scientific research documents, whether they are published or not. The documents may come from teaching and research institutions in France or abroad, or from public or private research centers.
L'archive ouverte pluridisciplinaire HAL, est destinée au dépôt et à la diffusion de documents scientifiques de niveau recherche, publiés ou non, émanant des établissements d'enseignement et de recherche français ou étrangers, des laboratoires publics ou privés. 

by frequency-shifted feedback

M. Guionie ${ }^{1}$, A. Thorette ${ }^{1}$, M. Romanelli ${ }^{1}$, A. Carré ${ }^{1}$, G. Loas ${ }^{1}$, E. Pinsard ${ }^{2}$,

L. Lablonde ${ }^{2}$, B. Cadier ${ }^{2}$, M. Alouini ${ }^{1}$, M. Vallet $^{1}$ and M. Brunel ${ }^{1}$

${ }^{1}$ Univ Rennes, CNRS, Institut FOTON - UMR 6082, Rennes ${ }^{2}$ iXblue Photonics, Lannion

Institut Foton, UMR 6082 CNRS, Université de Rennes 1, INSA Rennes, Lannion - France

Distributed-feedback (DFB) fiber lasers can sustain the oscillation of two orthogonal polarizations[1]. Such dual-frequency fiber lasers could be used as sensors or as a microwave optical source.

$\longrightarrow$ Phase stabilization needed for microwave photonics applications.

Solutions ? OPLL[2] but uses pump power as the actuator.

$\square$ Frequency-shifted feedback (FSF) as in bulk lasers[3]?

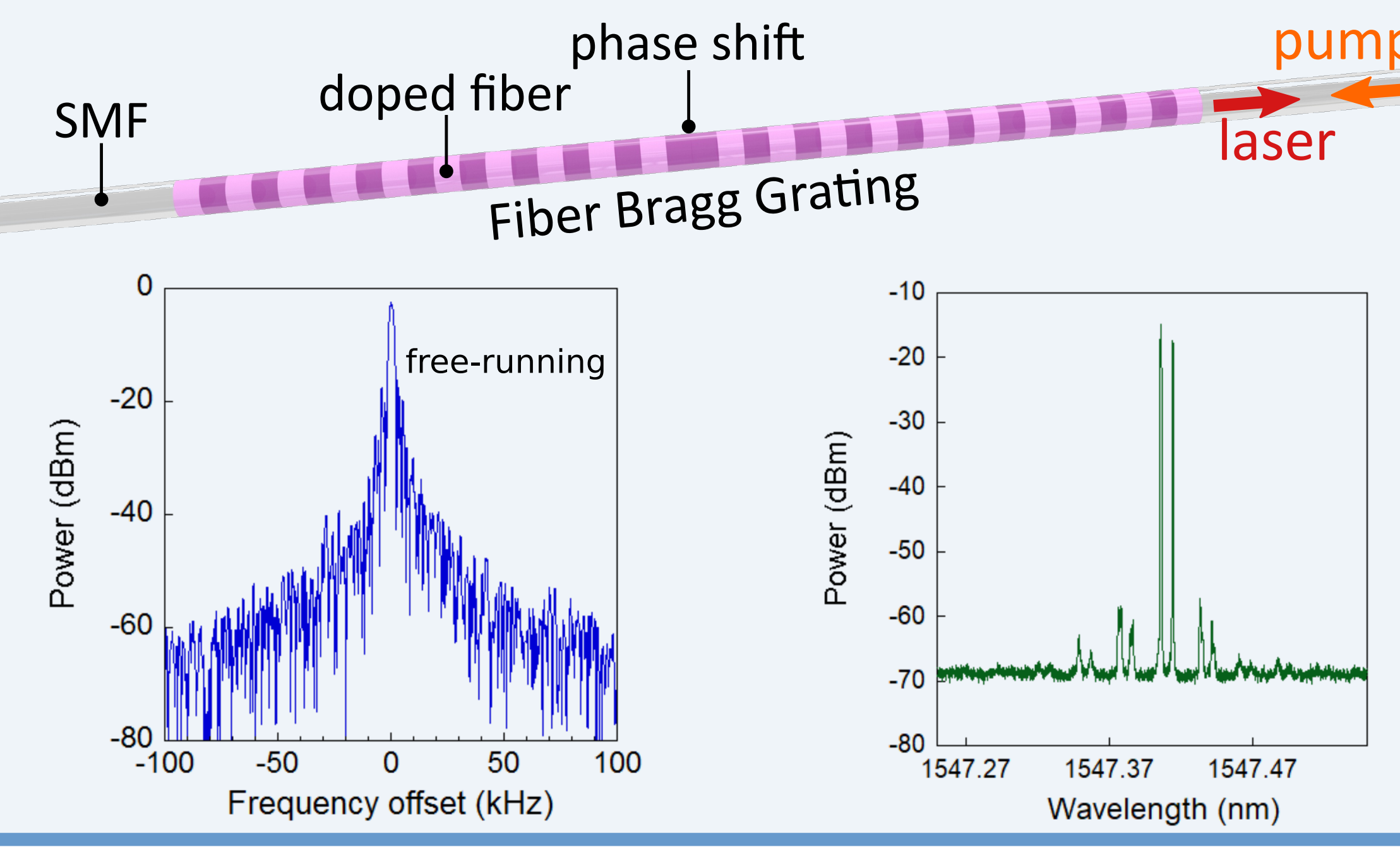

Stabilization on an external reference using FSF

One laser mode $(x)$ is re-injected

in the laser after frequency shift and polarization rotation

Detuning: $\Delta$

$\Delta=\nu_{y}-\left(\nu_{x}+f_{L O}\right)=f_{b}-f_{L O}$

Reinjection rate: $\Gamma$

Frequency locked if $\Delta<\Gamma$
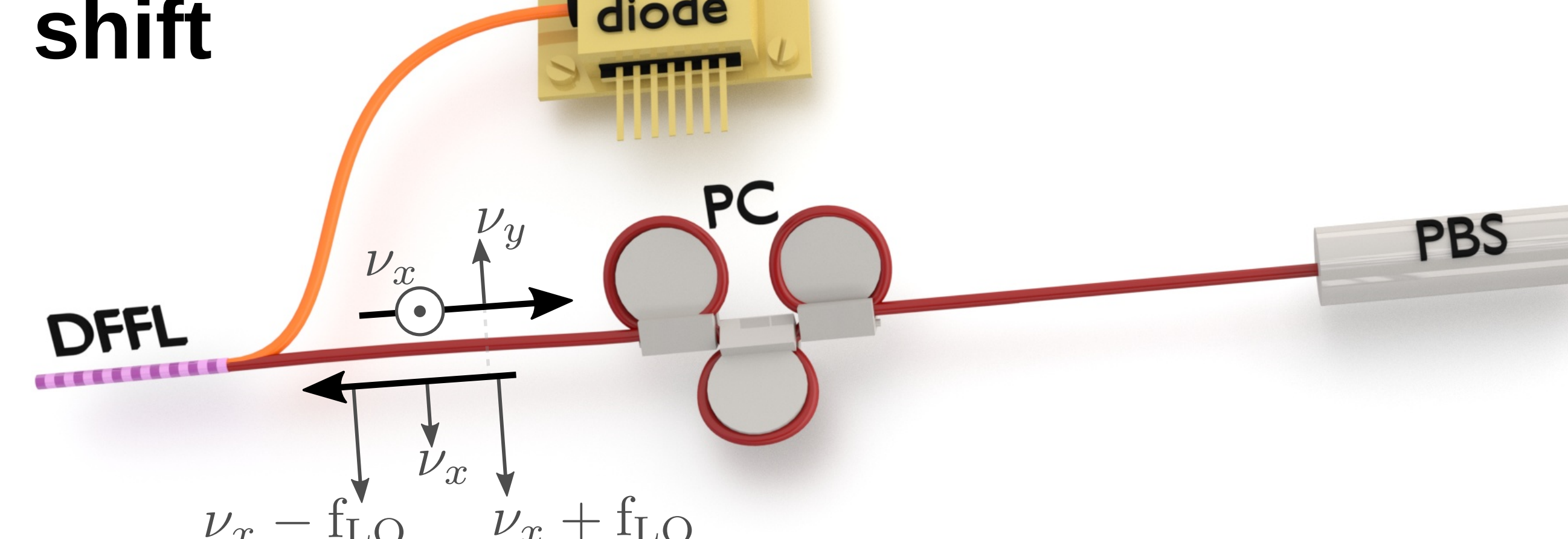
Exp: Locking range $4 \mathrm{MHz}$ when $\Gamma=3.010^{-4}$

Results for two different DFBs
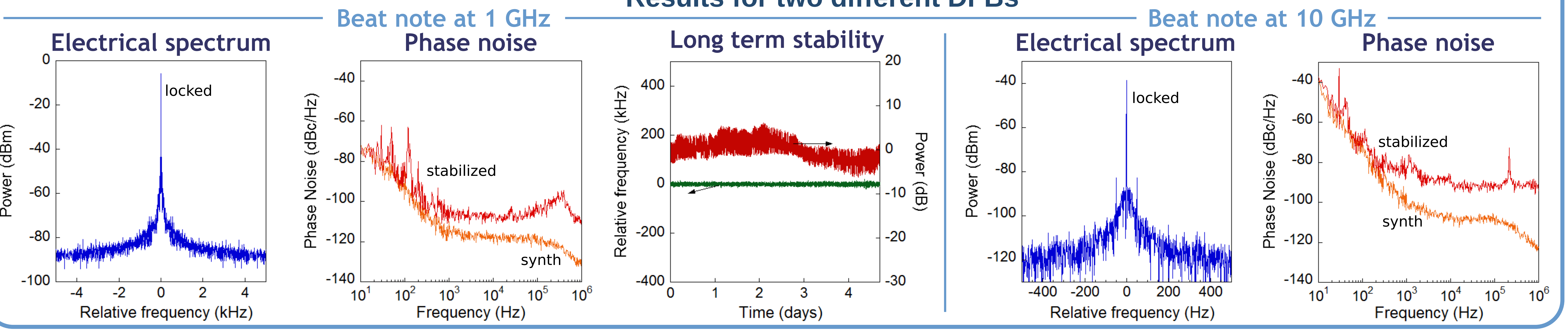

Self-stabilization using FSF and a delay-line Hybrid opto-electronic (OEO) scheme[4]

Dynamics governed by:

\begin{tabular}{|l|l|l|}
\hline Relaxation oscillation & $\tau_{\mathrm{r}} \approx 5 \mu \mathrm{s}$ & $200 \mathrm{kHz}$ \\
\hline FSF loop & $\tau=50 \mathrm{~ns}$ & $20 \mathrm{MHz}$ \\
\hline OEO delay line & $\tau_{\text {EO }}=25 \mu \mathrm{s}$ & $40 \mathrm{kHz}$ \\
\hline
\end{tabular}

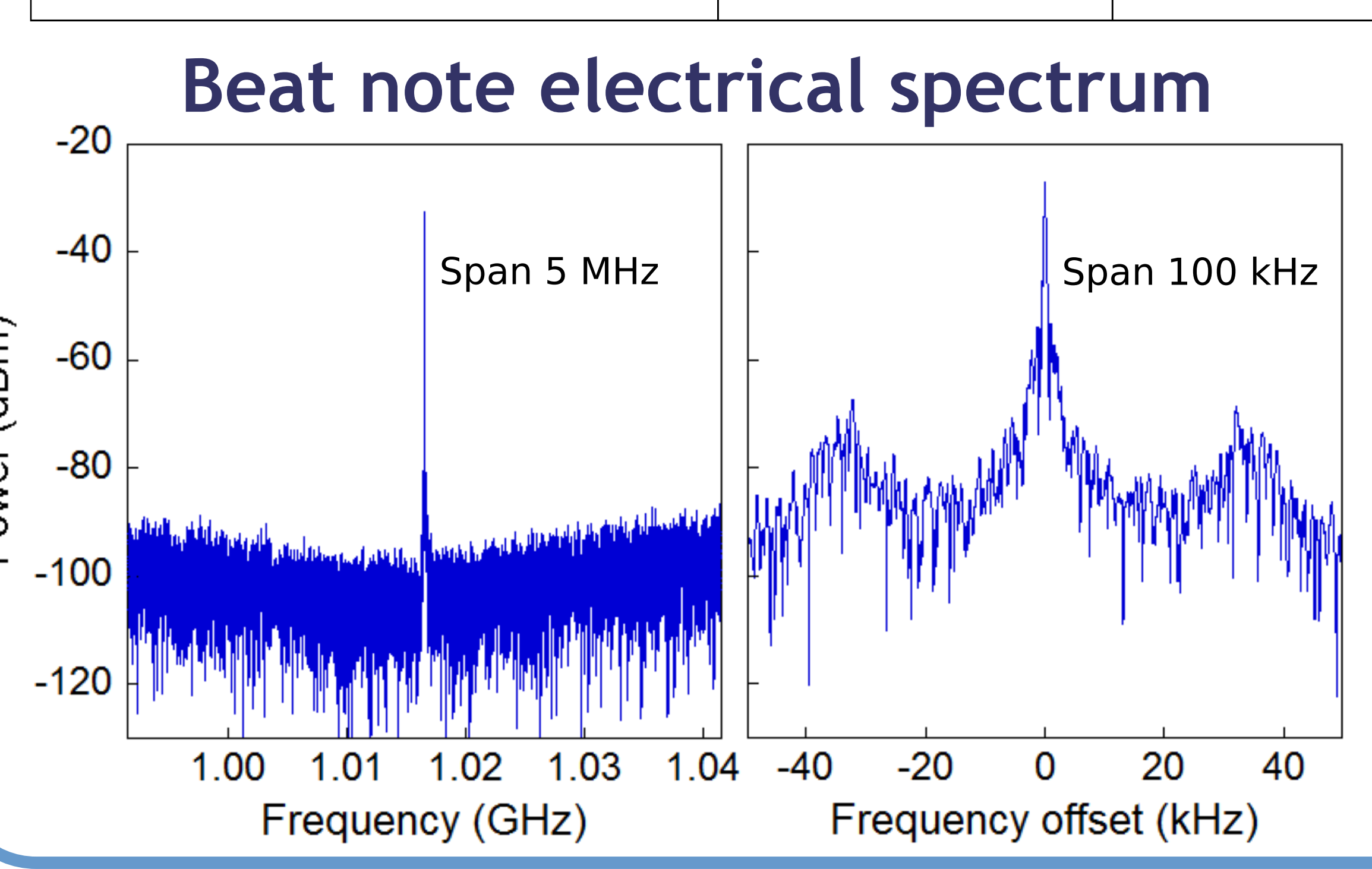

\section{Conclusions}

o Proof-of-principle of both methods validated - Methods are independent of the carrier (within limit of EOM bandwidth) - Reduction of linewidth (at least 100 times) and phase noise (better results on external reference than in the OPLL case)

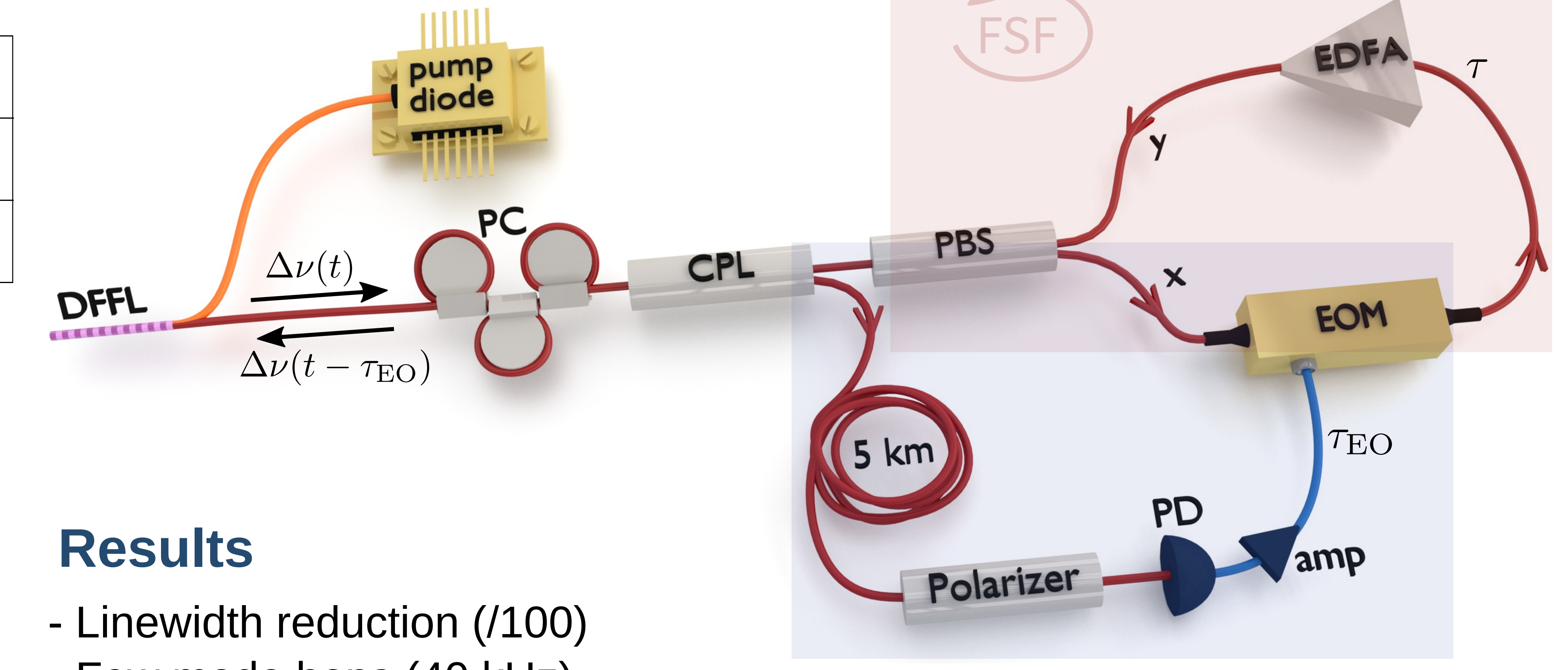

- Few mode hops (40 kHz)

- No RF filter needed (20 MHz resonances filtered by the laser)
Optical spectrum

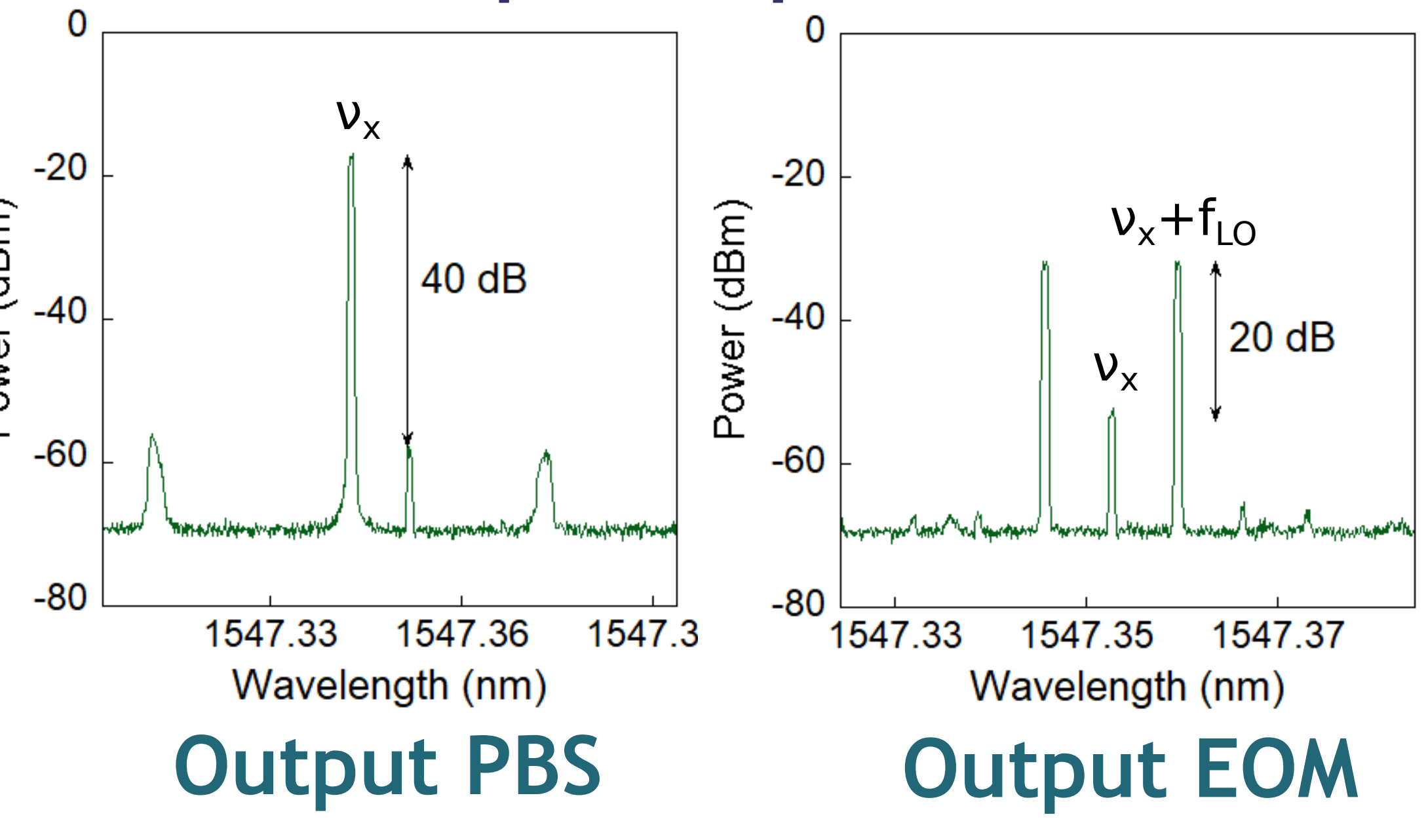

Funding DGA (ANR-16-ASTR-0016); Région Bretagne, FEDER, Rennes Metropole (CPER SOPHIE- Photonique). 